\title{
High performance optical filters based on advanced coupled Sagnac loop waveguide reflectors
}

\author{
Hamed Arianfard, Jiayang Wu, Saulius Juodkazis, and David J. Moss* \\ Optical Sciences Centre, Swinburne University of Technology, Hawthorn, VIC 3122, Australia.
}

\begin{abstract}
We present theoretical designs of high performance optical filters in integrated silicon photonic nanowire resonators. We use mode interference in formed by zig-zag waveguide coupled Sagnac loop reflectors (ZWC-SLRs), tailored to achieve diverse filtering functions with good performance. These include compact bandpass filters with improved roll-off, optical analogues of Fano resonances with ultrahigh spectral extinction ratios (ERs) and slope rates, and resonance mode splitting with high ERs and low free spectral ranges. The analysis verifies the feasibility of multi-functional integrated photonic filters based on ZWC-SLR resonators for flexible spectral engineering in diverse applications.
\end{abstract}

Keywords: Integrated optics, resonators, Fano resonance, mode splitting, classical filters.

\section{INTRODUCTION}

Micro/Nano-scale integrated photonic resonators enable a wide variety of optical functions in photonic integrated circuits [1-6]. Optical bandpass filters (BPFs) are core components in wavelength division multiplexing (WDM) optical communication systems [7]. To date, various types of photonic resonators have been designed to to improve the roll-off of optical filters for achieving quasi flat-top spectral responses, which are highly desirable in WDM optical communication systems $[8,9]$. However, these structures usually achieve flat-top spectral responses via cascading many subunits. This results in a bulky device footprint and, in addition, it is challenging to maintain the desired spectral response given the unequal wavelength drifts for different sub-components induced by the thermo-optic effect [10].

Fano resonances are fundamental physical phenomena exhibiting a distinctly asymmetric resonant lineshape profile arose from the constructive and destructive interference of a narrow discrete resonance with a broad spectral line or continuum [11-13]. Optical analogues of Fano resonances have been hot research topics in recent years and have found many applications[11, 14].

At the same time, resonance mode splitting is also a fundamental phenomenon in photonic resonators that occurs when two or more mutually coupled modes co-exist in the same resonant cavity $[15,16]$. Recently, many applications based on mode-split resonators have been introduced due to their capability of providing a reduced free spectral range (FSR) and an increased quality (Q) factor while maintaining a small physical cavity length [17-19]. This yields a compact device footprint, low power consumption, and versatile filter shapes for dense WDM (DWDM) and microwave photonics applications [20, 21].

Recently, we demonstrated multi-functional integrated photonic filters based on cascaded Sagnac loop reflectors (SLRs) [22] formed by self-coupled silicon-on-insulator (SOI) nanowires. Here, we theoretically investigate advanced filter structures based on zig-zag waveguide coupled Sagnac loop reflectors (ZWC-SLR) [23, 24], that yield greatly enhanced performance together with additional versatile filtering functions. The ZWC-SLR resonators can be considered to be hybrid filters consisting of both finite-impulse-response (FIR) and infinite-impulse-response (IIR) filter elements as well as standing-wave (SW) and travelling-wave (TW) filter elements that provide more versatile mode interference and significantly improved flexibility for spectral engineering [25-30]. Combining this approach with highly nonlinear thin films [30-47] would offer exciting possibilities for advanced nonlinear integrated devices. 
*dmoss@swin.edu.au

\section{DEVICE CONFIGURATION}

The schematic configurations of two and three ZWC-SLR resonators are illustrated in Figs. 1(a) and (b), respectively. To model the ZWC-SLR resonators based on the scattering matrix method [22, 23, 25], the waveguide and coupler parameters are defined in Table 1. To simplify the comparison, we assume that the two and three SLRs are identical in each ZWC-SLR resonator, i.e., $L_{\mathrm{SLR} i}(i=1-3)=L_{\mathrm{SLR}}, t_{\mathrm{si}}(i=1-3)=t_{\mathrm{s}}$, and $t_{\mathrm{b} i}(i=1-3)=t_{\mathrm{b}}$.

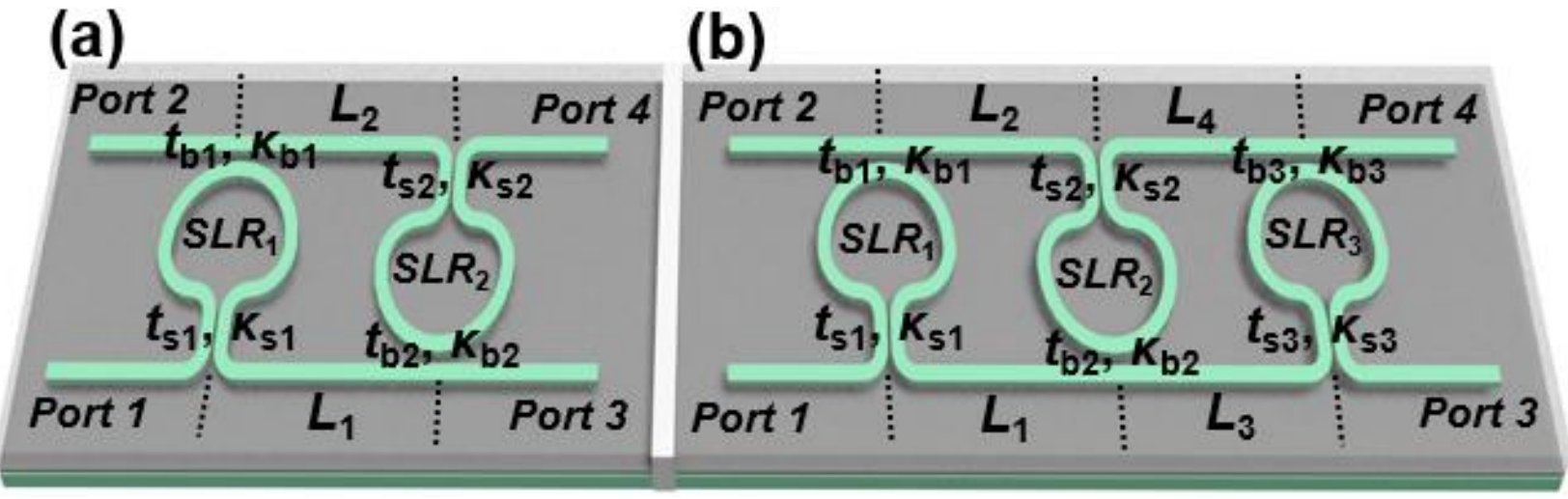

Figure 1. Schematic configuration of (a) two and (b) three ZWC-SLR resonators, respectively. The definitions of $t_{s i}(i=1,2$,

$3), t_{\mathrm{b} i}(i=1,2,3), L_{\mathrm{SLR} i}(i=1,2,3)$, and $L_{i}(i=1,2,3,4)$ are given in Table 1.

In the following sections, mode interference in the ZWC-SLR resonators is tailored to achieve high-performance filtering functions, including compact BPFs (Section 3), optical analogues of Fano resonances (Section 4), and resonance mode splitting (Section 5). In our design, we use values attained from our previously fabricated SOI devices [22, 26] for the waveguide group index $\left(n_{\mathrm{g}}=4.3350\right.$, transverse electric (TE) mode) and the propagation loss $\left(\alpha=55 \mathrm{~m}^{-1}\right.$, i.e., 2.4 $\mathrm{dB} / \mathrm{cm})$. The devices are designed based on but not limited to the SOI integrated platform.

Table 1. Definitions of structural parameters of the ZWC-SLR resonators

\begin{tabular}{|c|c|c|c|}
\hline Waveguides & Length & $\begin{array}{c}\text { Transmission } \\
\text { factor }^{\mathrm{a}}\end{array}$ & $\begin{array}{l}\text { Phase } \\
\text { shift b }\end{array}$ \\
\hline Bus waveguides between SLRs $(i=1,2,3,4)$ & $L_{i}$ & $a_{i}$ & $\varphi_{i}$ \\
\hline Sagnac loop in $\operatorname{SLR}_{i}(i=1,2,3)$ & $L_{\mathrm{SLR} i}$ & $a_{\mathrm{si}}$ & $\varphi_{s i}$ \\
\hline Directional couplers & Field transmission coefficient ${ }^{\mathrm{c}}$ & \multicolumn{2}{|c|}{ Field cross-coupling coefficient ${ }^{\mathrm{c}}$} \\
\hline Coupler in $\operatorname{SLR}_{i}(i=1,2,3)$ & $t_{\mathrm{si}}$ & \multicolumn{2}{|c|}{$k_{s i}$} \\
\hline Coupler between $S L R_{i}$ and bus waveguide $(i=1,2,3)$ & $t_{\mathrm{b} i}$ & \multicolumn{2}{|c|}{$k_{\mathrm{b} i}$} \\
\hline
\end{tabular}

${ }^{\mathrm{a}} a_{i}=\exp \left(-\alpha L_{i} / 2\right), a_{\mathrm{s} i}=\exp \left(-\alpha L_{\mathrm{SLR} i} / 2\right), \alpha$ is the power propagation loss factor.

${ }^{\mathrm{b}} \varphi_{i}=2 \pi n_{\mathrm{g}} L_{i} / \lambda, \varphi_{\mathrm{s} i}=2 \pi n_{\mathrm{g}} L_{\mathrm{SLR} i} / \lambda, n_{\mathrm{g}}$ is the group index and $\lambda$ is the wavelength.

${ }^{\mathrm{c}} t_{\mathrm{si}}{ }^{2}+\kappa_{\mathrm{s} i}{ }^{2}=1$ and $t_{\mathrm{bi}}{ }^{2}+\kappa \mathrm{b} i^{2}=1$ for lossless coupling are assumed for all the directional couplers.

\section{COMPACT BANDPASS FILTERS WITH IMPROVED ROLL-OFF}

In this section, we tailor the mode interference in the two ZWC-SLR resonator to realize compact BPFs with improved roll-off. Figures 2(a) and (b) show the power transmission spectrum and corresponding group delay response of the two ZWC -SLR resonator from Port 1 to Port 2 in the wavelength range of $1548.9 \mathrm{~nm}-1551.2 \mathrm{~nm}$, respectively. There are wide-flat stopbands and a passband with improved roll-off, arising from coherent mode interference within the two ZWC -SLR resonator. The structural parameters are $L_{\mathrm{SLR}}=L=100 \mu \mathrm{m}, t_{\mathrm{s}}=t_{\mathrm{b}}=0.78$.

To quantitatively analyze the improvement in the filtering roll-off, we further compare the 3-dB BW of the BPF based on two ZWC -SLRs (2-ZWC-SLRs) with BPFs considering other types of integrated photonic resonators, including a single add-drop MRR (1-MRR) [27, 28], two cascaded SLRs (2-C-SLRs) [29], three cascaded SLRs (3-C-SLRs) [22], and two parallel coupled MRRs (2-MRRs) [27, 28]. In comparison, the above filters were designed based on the same SOI wire 
waveguide (i.e., with the same $n_{\mathrm{g}}=4.3350$ and $\alpha=55 \mathrm{~m}^{-1}$ ). Figure 3(a) shows the normalized power transmission spectra of the BPFs considering the various types of integrated resonators mentioned above. The filtering spectra of all the
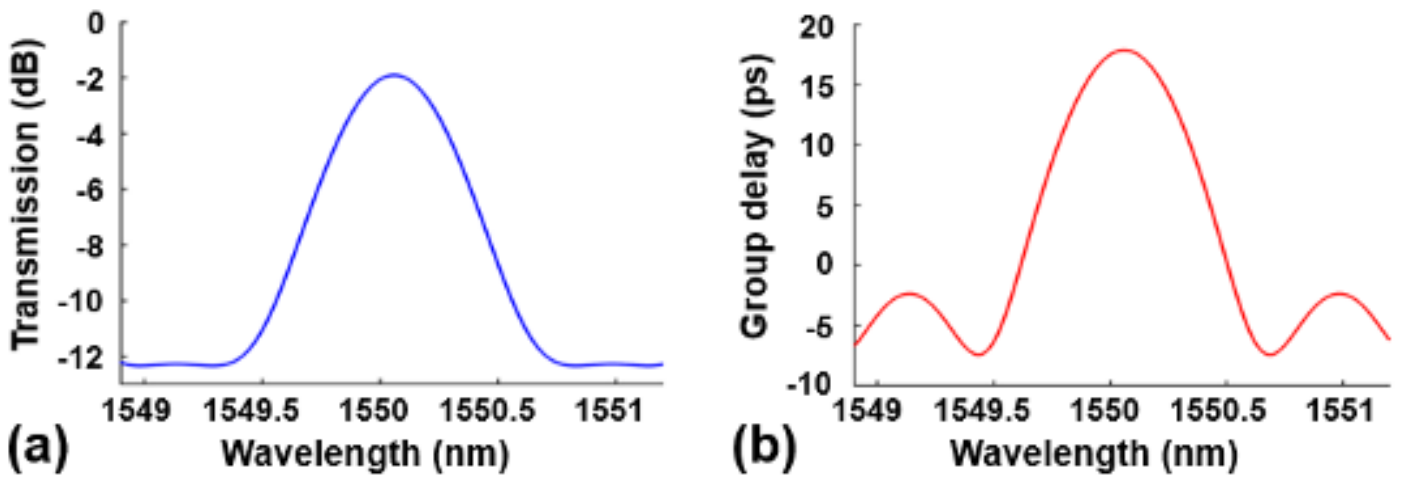

Figure 2. (a) Power transmission spectra of the two ZWC-SLR resonator from Port 1 to Port 2 in the wavelength range of $1548.9 \mathrm{~nm}-1551.2 \mathrm{~nm}$ when $L_{\mathrm{SLR}}=L=100 \mu \mathrm{m}, t_{\mathrm{s}}=t_{\mathrm{b}}=0.78$. (b) Group delay of the BPF in (a).

devices were normalized to have the same ER $(\sim 10.36 \mathrm{~dB})$ and full width at minimum $(\sim 230.6 \mathrm{GHz})$ as those of the BPF in Fig. 2(a). The corresponding 3-dB BWs are given in Fig. 3(b). It is clear that the BPF based on the two ZWC-SLRs resonator has the largest 3-dB BW and the best roll-off, reflecting enhanced mode interference in this compact device consisting of only two SLRs.
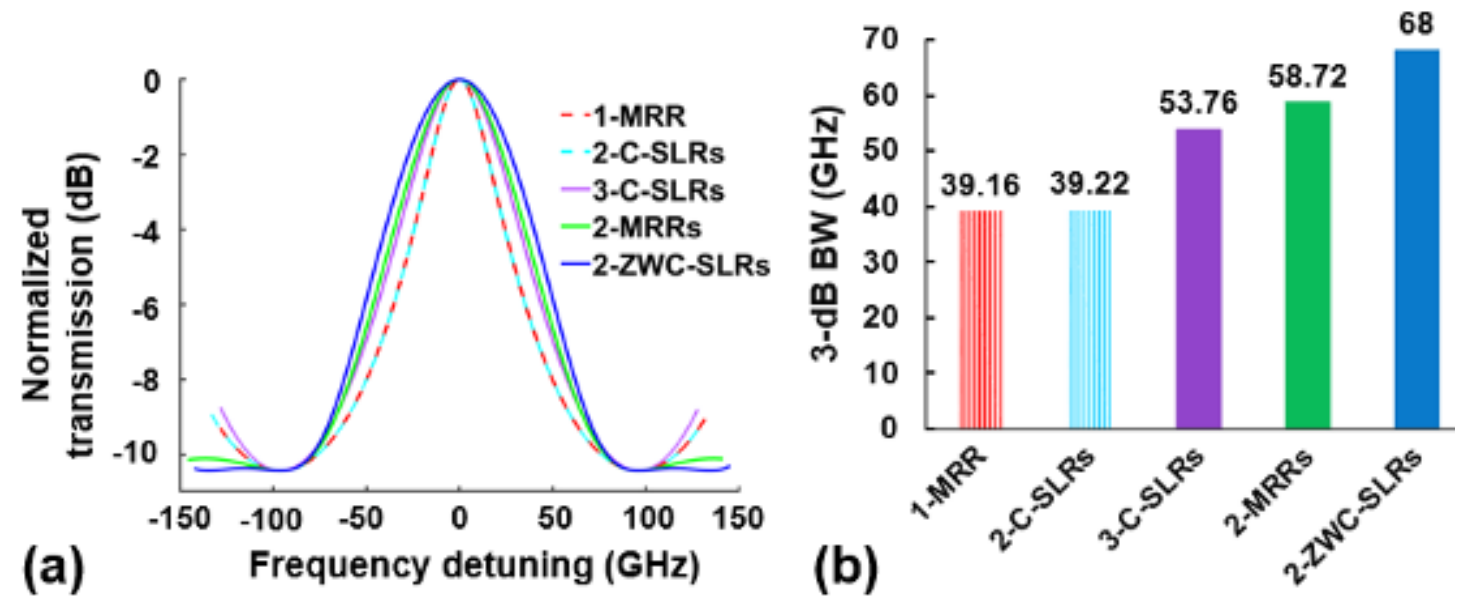

Figure 3. (a) Normalized transmission spectra of BPFs based on various types of integrated photonic resonators, including single add-drop MRR (1-MRR), two cascaded SLRs (2-C-SLRs), three cascaded SLRs (3-C-SLRs), two parallel coupled MRRs (2-MRRs) and two ZWC-SLRs (2-ZWC-SLRs). (b) 3-dB BWs of the BPFs in (a).

\section{ULTRA-SHARP FANO RESONANCES}

In this section, we tailor the spectral response of the three ZWC-SLR resonator structure to realize optical analogues of Fano resonances with high ERs and SRs. The power transmission spectrum from Port 2 to Port 4 of the three ZWC-SLR resonator is depicted in Fig. 4(a). One can see that there are periodical Fano resonances with identical asymmetric resonant line-shapes in each period. The device structural parameters are $L_{\mathrm{SLR}}=L_{1,2,3,4}=115 \mu \mathrm{m}, t_{\mathrm{s}}=0.743$, and $t_{\mathrm{b}}=$ 0.994. The FSR is about $200 \mathrm{GHz}$, which equals the sum of the two wavelength spacings (WS1 and WS2). The two WSs are very close to each other (WS1 $=101.71 \mathrm{GHz}$ and WS2 $=98.88 \mathrm{GHz}$ ), reflecting the high SR of the Fano resonances. Figure 4(b) shows a zoom-in view of Fig. 3(a) in the wavelength range of $1549.8 \mathrm{~nm}-1550.65 \mathrm{~nm}$, which shows a Fano resonance with an ultra-high ER of $76.32 \mathrm{~dB}$ and an ultra-high SR of $997.66 \mathrm{~dB} / \mathrm{nm}$. The ER is defined as the difference between the maximum and the minimum transmission, and the SR is defined as the ratio of the ER to the wavelength difference between the resonance peak and notch (i.e., $\Delta \lambda$ in Fig. 4(b)). The high ER and SR reflect the high performance of the Fano resonances resulting from strong coherent optical mode interference in the compact resonator 
with only three SLRs. Further, the periodical filter shape of the zig-zag 3WC-SLR resonator is also useful for applications in WDM systems.

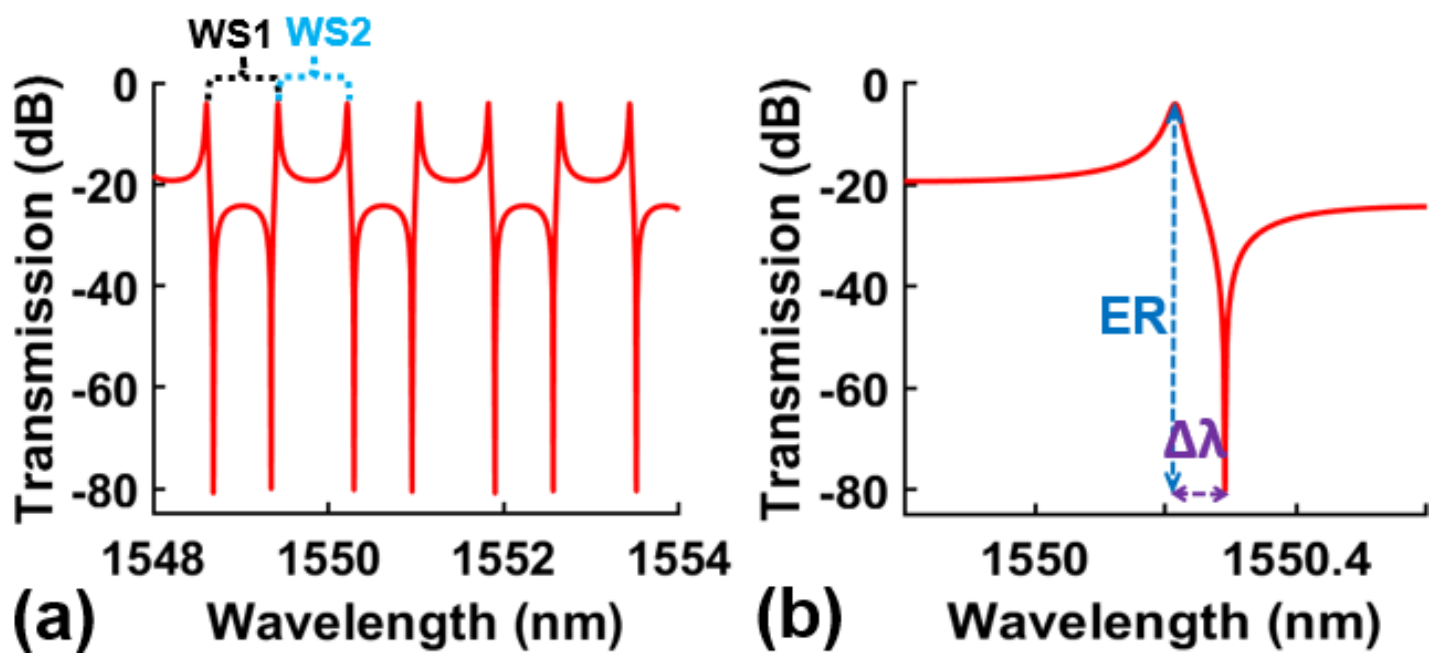

Figure. 4. (a) Power transmission spectrum of the three ZWC-SLR resonator from Port 2 to Port 4 when $L S L R=L_{1,2,3,4}=115$ $\mu \mathrm{m}, t_{\mathrm{s}}=0.743$, and $t_{\mathrm{b}}=0.994$. (b) Zoom-in view of (a) in the wavelength range of $1549.8 \mathrm{~nm}-1550.65 \mathrm{~nm}$. WS: wavelength spacing. ER: extinction ratio. $\Delta \lambda$ : wavelength difference between the resonance peak and notch.

Figure 5(a) compares the power transmission spectra for various $t_{\mathrm{s}}$ (reflectivity of SLRs). we changed only $t_{\mathrm{s}}$, keeping the other structural parameter the same as those in Fig. 4. The corresponding IL and SR are depicted in Fig. 5(b). The IL increases with $t_{\mathrm{s}}$, while the SR first increases and then decreases with $t_{\mathrm{s}}$, achieving a maximum value of $997.66 \mathrm{~dB} / \mathrm{nm}$ at $t_{\mathrm{s}}=0.743$. The non-monotonic relationship between the SR and $t_{\mathrm{s}}$ is a combined result of both a decrease in $\Delta \lambda$ and a non-monotonic variation in ER. The latter mainly arises from the difference between the internal (transmission) and external (coupling) cavity loss, which is similar to that for different coupling regimes in microring resonators (MRRs) [30].
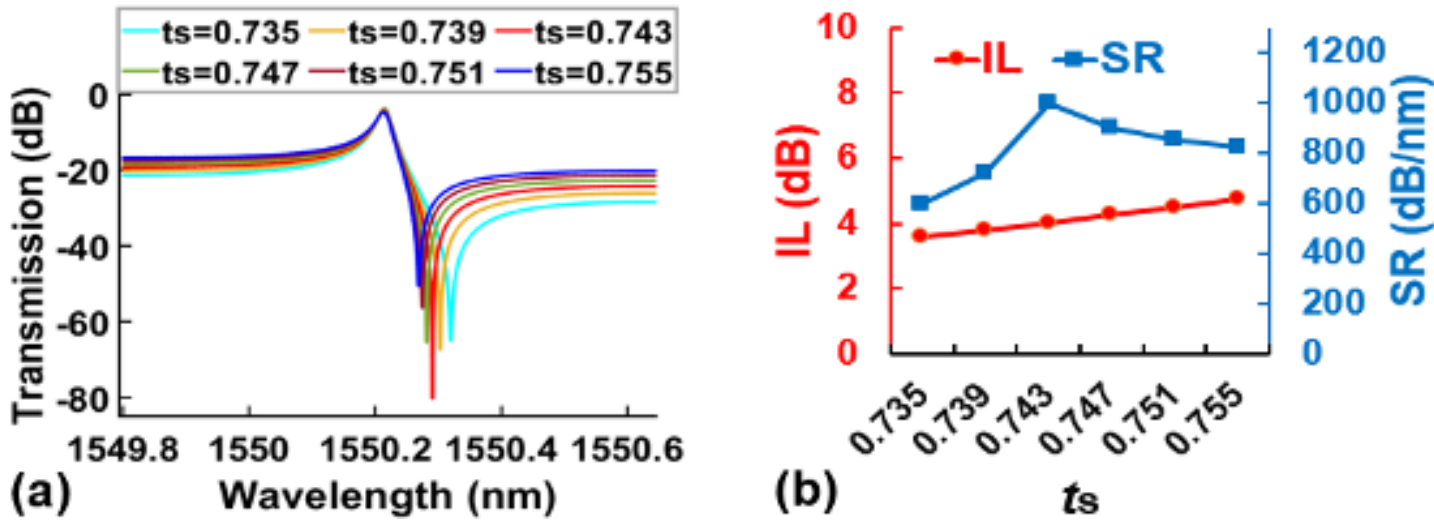

Figure. 5. (a) Power transmission spectra and (b) the corresponding IL and SR for various $t_{\mathrm{s}}$ when $t_{\mathrm{b}}=0.994$ and $L_{\mathrm{SLR}}=L_{1,2}$, $3,4=115 \mu \mathrm{m}$, respectively.

\section{RESONANCE MODE SPLITTING}

In this section, we tailor the mode interference in the three ZWC-SLR resonator to achieve resonance mode splitting with high ERs and low FSRs. The resonance mode splitting with multiple densely spaced resonances can break the dependence between the Q factor, FSR, and physical cavity length, thus allowing low FSRs and high Q factors in resonators with a compact footprint. Figure 6(a) shows the power transmission spectrum from Port 2 to Port 4 of the three ZWC-SLR resonator. The structural parameters are $L_{\mathrm{SLR}}=L_{1,2,3,4}=115 \mu \mathrm{m}, t_{\mathrm{s}}=0.72$, and $t_{\mathrm{b}}=0.99$, which are designed in order to achieve a WS of about $100 \mathrm{GHz}$ between adjacent split resonances. In Fig. 6(a), WS1 $=98.33 \mathrm{GHz}$ 
and WS2 $=102.26 \mathrm{GHz}$. There are two split resonances within a FSR of $\sim 200.59 \mathrm{GHz}$. Figure 6(b) shows a zoom-in view of Fig. 6(a) in the wavelength range of $1549 \mathrm{~nm}-1550.7 \mathrm{~nm}$. The IL, Q factor, ER1, and ER2 of the two split resonances in Fig. 4(b) are $\sim 2.02 \mathrm{~dB}, \sim 6.03 \times 10^{4}, \sim 24.65 \mathrm{~dB}$, and $\sim 27.55 \mathrm{~dB}$, respectively.

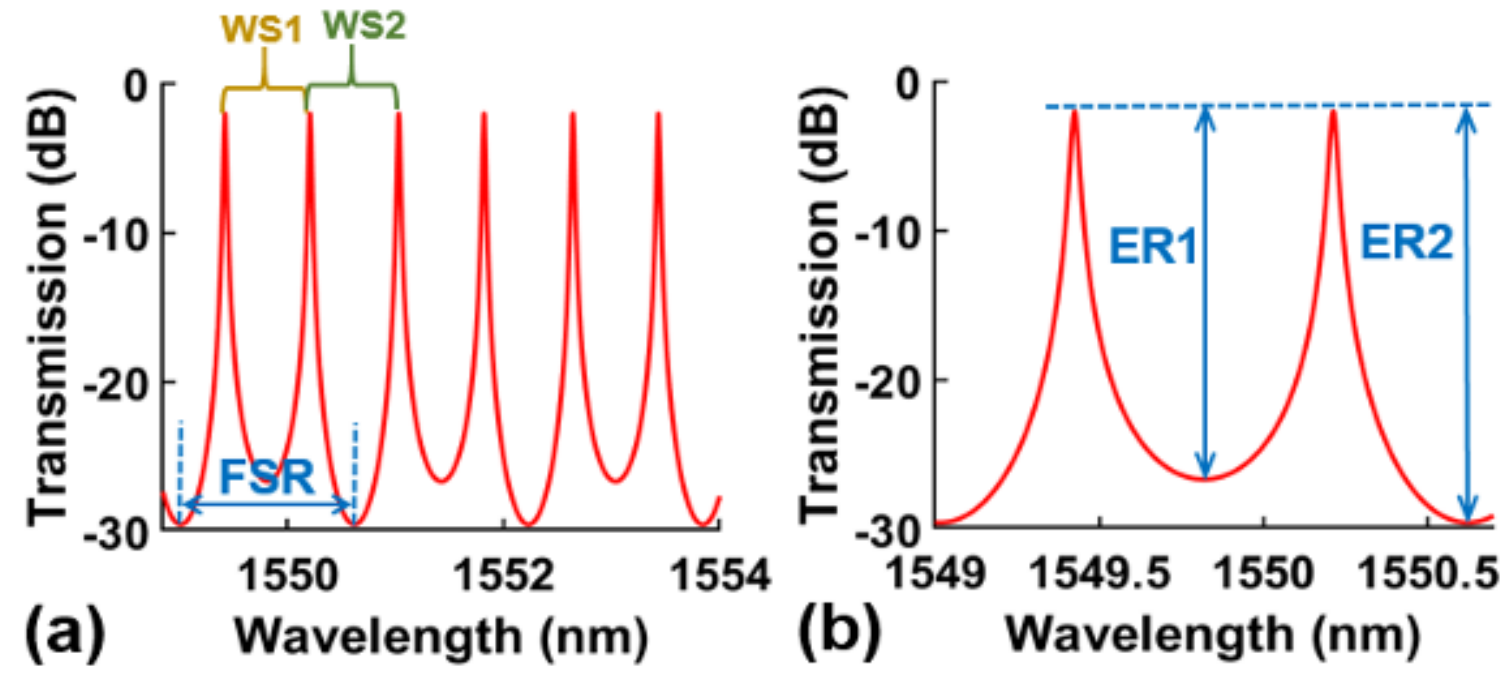

Figure 6. (a) Power transmission spectrum of the three ZWC-SLR resonator from Port 2 to Port 4 when $L$ SLR $=L_{1,2,3,4}=115$ $\mu \mathrm{m}, t_{\mathrm{s}}=0.72$, and $t_{\mathrm{b}}=0.99$. (b) Zoom-in view of (a) in the wavelength range of $1549 \mathrm{~nm}-1550.7 \mathrm{~nm}$.

Figure 7(a) shows the spectral response for various $t_{\mathrm{s}}$. we only changed the reflectivity of SLRs, keeping the other structural parameters the same as those in Fig. 6 (a). The Q factor and ERs (ER1 and ER2) as functions of $t_{\mathrm{s}}$ are depicted in Fig. 7(b). As $t_{\mathrm{s}}$ increases, the Q factor slightly decreases while the ER1 and ER2 change more dramatically, resulting in a change in the spectral response towards that of the Fano resonances in Fig. 4(a). The non-monotonic change in ER2 with $t_{\mathrm{s}}$ follows the trend of the SR in Fig. 5(b) for similar reasons. In particular, ER1 equals to ER2 when $t_{\mathrm{s}}=0.7177$. Under this condition, the $\mathrm{Q}$ factor and effective FSR are $\sim 6.06 \times 10^{4}$ and $\sim 100.30$ GHz (i.e., half of the FSR in Fig. 6(a)), respectively.
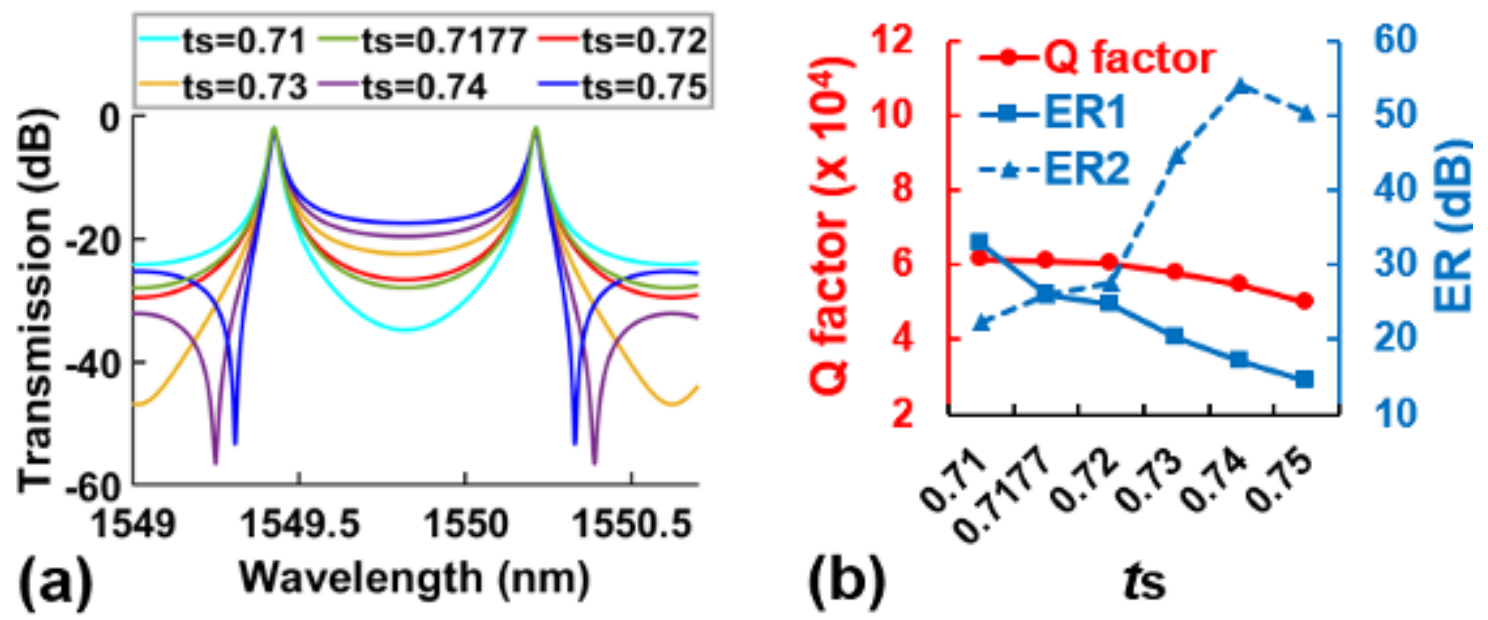

Figure 7. (a) Power transmission spectra of the three ZWC-SLR resonator for various $t_{\mathrm{s}}$ for input from Port 2 to Port 4 when $t_{\mathrm{b}}=0.99$ and $L_{\mathrm{SLR}}=L_{1,2,3,4}=115 \mu \mathrm{m}$. (b) Calculated Q factor and ERs (ER1 and ER2) as functions of $t_{\mathrm{s}}$ for the transmission spectra in (a).

To achieve the same FSR, the circumference of a comparable MRR (with the same waveguide geometry and loss) is 690 $\mu \mathrm{m}$, which is 6 times the length of the SLRs. This highlights the reduced cavity length enabled by the mode splitting in the 3WC-SLR resonator. On the other hand, the Q factor of a comparable MRR with the same FSR and ER is $\sim 6.08 \times$ $10^{4}$ - almost the same as that of the zig-zag $3 \mathrm{WC}$-SLR resonator. This indicates that the reduced cavity length did not come at the expense of a significant decrease in $\mathrm{Q}$ factor. 
The number of split resonances can be changed by varying the length of the connecting bus waveguides. Figure 8(a) shows the power transmission spectrum from Port 1 to Port 3 of the three ZWC-SLR resonator. Clearly, there are four split resonances in each FSR. The structural parameters are $L_{\mathrm{SLR}}=115 \mu \mathrm{m}, L_{1,3}=115 \mu \mathrm{m}, L_{2,4}=230 \mu \mathrm{m}$, and $t_{\mathrm{s}}=t_{\mathrm{b}}=$ 0.88. The WSs between the split resonances are WS1 $=\mathrm{WS} 3=100.46 \mathrm{GHz}$ and WS2 $=90.37 \mathrm{GHz}$. Figure 8 (b) shows a zoom-in view of Fig. 8(a) in the wavelength range of $1548.7 \mathrm{~nm}-1550.7 \mathrm{~nm}$.

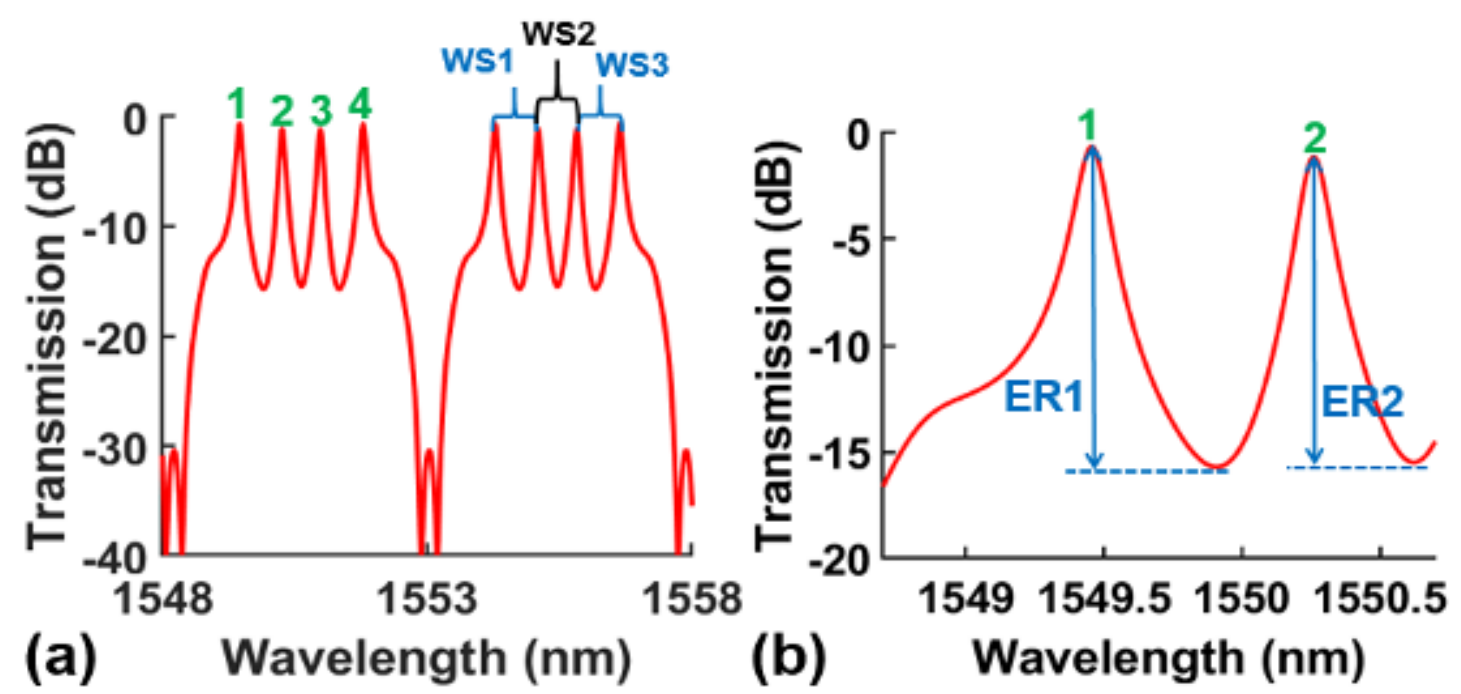

Figure 8. (a) Power transmission spectrum of the three ZWC-SLR resonator from Port 1 to Port 3 when $L_{S L R}=115 \mu \mathrm{m}, L_{1,3}$ $=115 \mu \mathrm{m}, L_{2,4}=230 \mu \mathrm{m}$, and $t_{\mathrm{s}}=t_{\mathrm{b}}=0.88$. (b) Zoom-in view of (a) in the wavelength range of $1548.7 \mathrm{~nm}-1550.7 \mathrm{~nm}$.

The power transmission spectra for different $t_{\mathrm{s}}$ is shown in Fig. 9(a). The corresponding Q factors (Q1 and Q2) and ERs (ER1 and ER2) for the first two resonances from the left side are shown in Fig. 9(b). In Figs. 9(a) and (b), all the Q factors and ERs decrease with $t_{\mathrm{s}}$, along with slightly decreased ILs.
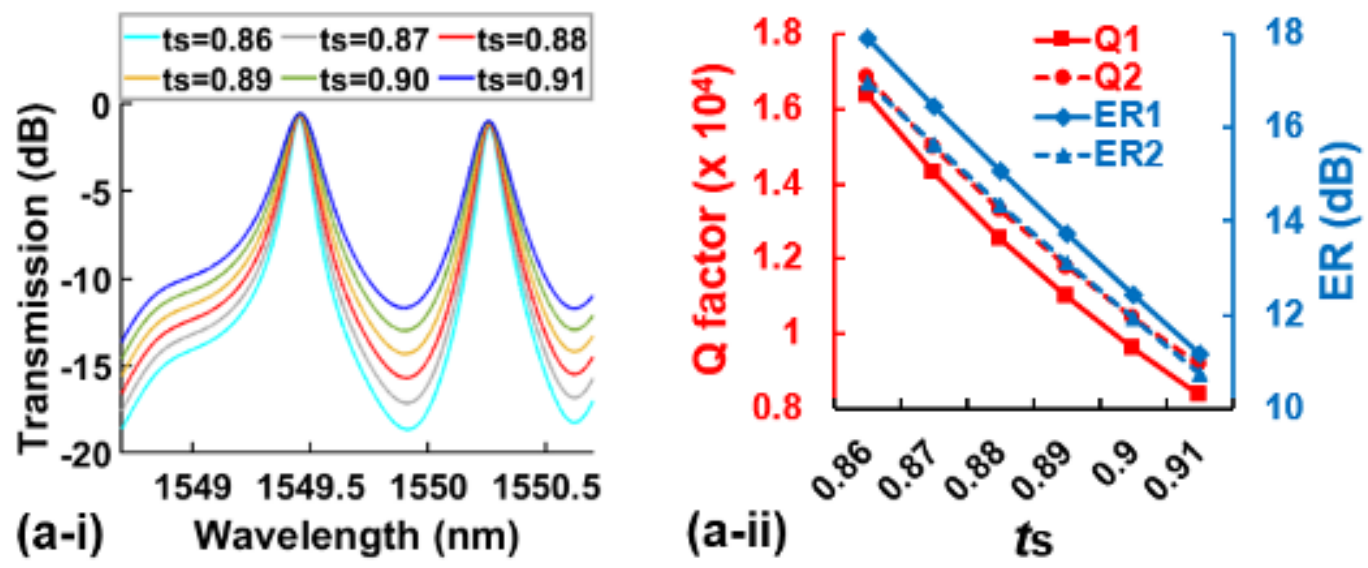

Figure 9. (a) Power transmission spectra of the three ZWC-SLR resonator for various $t_{\mathrm{s}}$ for input from Port 1 to Port 3 when $t_{\mathrm{b}}=0.88, L_{\mathrm{SLR}}=115 \mu \mathrm{m}, L_{1,3}=115 \mu \mathrm{m}$, and $L_{2,4}=230 \mu \mathrm{m}$. (b) Calculated Q factors (Q1 and Q2) and ERs (ER1 and ER2) as functions of $t_{\mathrm{s}}$ for the transmission spectra in (a).

\section{CONCLUSIONS}

We theoretically investigate advanced multi-functional integrated photonic filters based on ZWC-SLR resonators. Mode interference in the ZWC-SLR resonators is tailored to achieve different filtering functions including compact bandpass filters with improved roll-off, optical analogues of Fano resonances with ultrahigh ERs and SRs, resonance mode splitting with high ERs and low FSRs. This work highlights the ZWC-SLR resonators as a robust and adaptable approach to flexible spectral engineering for a diverse range of applications. 


\section{REFRENCES}

[1] W. Bogaerts, P. De Heyn, T. Van Vaerenbergh et al., "Silicon microring resonators," Laser \& Photonics Reviews, 6(1), 47-73 (2012).

[2] H. Arianfard, B. Khajeheian, and R. Ghayour, "Tunable band (pass and stop) filters based on plasmonic structures using Kerr-type nonlinear rectangular nanocavity," Optical Engineering, 56(12), 121902 (2016).

[3] Z. Yao, K. Wu, B. X. Tan et al., "Integrated silicon photonic microresonators: emerging technologies," IEEE Journal of Selected Topics in Quantum Electronics, 24(6), 1-24 (2018).

[4] J. Wu, Y. Yang, Y. Qu et al., "2D layered graphene oxide films integrated with micro-ring resonators for enhanced nonlinear optics," Small, e1906563 (2020).

[5] H. Arianfard, and R. Ghayour, "Nanoscale plasmonic filter based on coupled metal-insulator-metal waveguides using nonlinear nanoslot resonator," Journal of Nanophotonics, 9(1), 093799 (2014).

[6] J. Wu, P. Cao, X. Hu et al., "Compact tunable silicon photonic differential-equation solver for general linear time-invariant systems," Opt Express, 22(21), 26254-64 (2014).

[7] C. M. Weinert, [Optical Filters in Wavelength-Division Multiplex Systems] Springer Berlin Heidelberg, Berlin, Heidelberg(2006).

[8] T. Dai, A. Shen, G. Wang et al., "Bandwidth and wavelength tunable optical passband filter based on silicon multiple microring resonators," Optics Letters, 41(20), 4807-4810 (2016).

[9] B. E. Little, S. T. Chu, P. P. Absil et al., "Very high-order microring resonator filters for WDM applications," IEEE Photonics Technology Letters, 16(10), 2263-2265 (2004).

[10] J. Wu, B. Liu, J. Peng et al., "On-Chip tunable second-order differential-equation solver based on a silicon photonic mode-split microresonator," Journal of Lightwave Technology, $\underline{\mathbf{3 3}}$ (17), 3542-3549 (2015).

[11] M. F. Limonov, M. V. Rybin, A. N. Poddubny et al., "Fano resonances in photonics," Nature Photonics, 11, 543 (2017).

[12] J. Chen, F. Gan, Y. Wang et al., "Plasmonic sensing and modulation based on Fano resonances," Advanced Optical Materials, 6(9), 1701152 (2018).

[13] B. Luk'yanchuk, N. I. Zheludev, S. A. Maier et al., "The Fano resonance in plasmonic nanostructures and metamaterials," Nature Materials, 9(9), 707-715 (2010).

[14] E. Kamenetskii, A. Sadreev, and A. Miroshnichenko, [Fano Resonances in Optics and Microwaves: Physics and Applications] Springer International Publishing, (2018).

[15] B. Peng, Ş. K. Özdemir, W. Chen et al., "What is and what is not electromagnetically induced transparency in whispering-gallery microcavities," Nature Communications, 5(1), 5082 (2014).

[16] Q. Li, T. Wang, Y. Su et al., "Coupled mode theory analysis of mode-splitting in coupled cavity system," Optics Express, 18(8), 8367-8382 (2010).

[17] Q. Li, Z. Zhang, F. Liu et al., "Dense wavelength conversion and multicasting in a resonance-split silicon microring," Applied Physics Letters, 93(8), 081113 (2008).

[18] J. Zhu, S. K. Ozdemir, Y.-F. Xiao et al., "On-chip single nanoparticle detection and sizing by mode splitting in an ultrahigh-Q microresonator," Nature Photonics, 4(1), 46-49 (2010).

[19] J. Wu, J. Peng, B. Liu et al., "Passive silicon photonic devices for microwave photonic signal processing," Optics Communications, $\underline{373}$, 44-52 (2016).

[20] M. C. Souza, G. F. Rezende, L. A. Barea et al., "Spectral engineering with coupled microcavities: active control of resonant mode-splitting," Opt Lett, 40(14), 3332-5 (2015).

[21] L. A. M. Barea, F. Vallini, P. F. Jarschel et al., "Silicon technology compatible photonic molecules for compact optical signal processing," Applied Physics Letters, 103(20), 201102 (2013).

[22] J. Wu, T. Moein, X. Xu et al., "Advanced photonic filters based on cascaded Sagnac loop reflector resonators in silicon-on-insulator nanowires," APL Photonics, Vol. 3, No. 4, 046102 (2018). DOI:10.1063/1.5025833.

[23] H. Arianfard, J. Wu, S. Juodkazis et al., "Advanced multi-functional integrated photonic filters based on coupled Sagnac loop reflectors," Journal of Lightwave Technology, Early Access, 1-9 (2020). doi: 10.1109/JLT.2020.3037559.

[24] H. Arianfard, J. Wu, S. Juodkazis, and D.J.Moss, "Three coupled waveguide Sagnac loop reflectors for high performance integrated optical filters," TechRxiv. Preprint. (2020). DOI: 10.36227/techrxiv.13301474.v1.

[25] J. Wu, P. Cao, T. Pan et al., "Compact on-chip $1 \times 2$ wavelength selective switch based on silicon microring resonator with nested pairs of subrings," Photonics Research, $\underline{\mathbf{3}}(1)$, 9-14 (2015).

[26] J. Wu, T. Moein, X. Xu et al., "Micro-ring resonator quality factor enhancement via an integrated Fabry-Perot cavity," APL Photonics, Vol. 2, No. 5, 056103 (2017). doi: 10.1063/1.4981392. 
[27] A. Jian, L. Zou, H. Tang et al., "Theoretical analysis of microring resonator-based biosensor with high resolution and free of temperature influence," Optical Engineering, 56(6), 067103 (2017).

[28] M. Borghi, A. Trenti, and L. Pavesi, "Four wave mixing control in a photonic molecule made by silicon microring resonators," Scientific Reports, 9(1), 408 (2019).

[29] X. Sun, L. Zhou, J. Xie et al., "Tunable silicon Fabry-Perot comb filters formed by Sagnac loop mirrors," Optics Letters, 38(4), 567-569 (2013).

[30] J. Wu, P. Cao, X. Hu et al., "Nested configuration of silicon microring resonator with multiple coupling regimes," IEEE Photonics Technology Letters, 25(6), 580-583 (2013).

[31] Yuning Zhang, Jiayang Wu, Yang Qu, Linnan Jia, Baohua Jia, and David J. Moss, "Design of silicon waveguides integrated with 2D graphene oxide films for Kerr nonlinear optics", TechRxiv. Preprint. (2020). https://doi.org/10.36227/techrxiv.13203278.v1.

[32] Yang Qu, Jiayang Wu, Yuning Zhang, Yao Liang, Baohua Jia, and David J. Moss, "Design analysis of fourwave-mixing in SiN nanowires integrated with graphene oxide films", TechRxiv. Preprint. (2020). https://doi.org/10.36227/techrxiv.13147622.v1.

[33] Jiayang Wu, Linnan Jia, Yuning Zhang, Yang Qu, Baohua Jia, and David J. Moss, “ Graphene oxide: versatile films for flat optics to nonlinear photonic chips”, Advanced Materials $\underline{32}$ 2006415, pp.1-29 (2020). DOI:10.1002/adma.202006415.

[34] Y. Qu, J. Wu, Y. Yang, Y. Zhang, Y. Liang, H. El Dirani, R. Crochemore, P. Demongodin, C. Sciancalepore, C. Grillet, C. Monat, B.aohua Jia, and D. J. Moss, "Enhanced nonlinear four-wave mixing in silicon nitride waveguides integrated with 2D layered graphene oxide films", Advanced Optical Materials $\underline{\mathbf{8}}$ (21) 2001048 (2020). DOI: 10.1002/adom.202001048.

[35] Linnan Jia, Jiayang Wu, Yunyi Yang, Yi Du, Baohua Jia, David J. Moss, "Large Third-Order Optical Kerr Nonlinearity in Nanometer-Thick PdSe 2 2D Dichalcogenide Films: Implications for Nonlinear Photonic Devices", ACS Applied Nano Materials $\underline{\mathbf{3}}$ (7) 6876-6883 June 29 (2020). DOI:10.1021/acsanm.0c01239.

[36] Yuning Zhang, Yang Qu, Jiayang Wu, Linnan Jia, Yunyi Yang, Xingyuan Xu, Baohua Jia, and David J. Moss, "Enhanced Kerr nonlinearity and nonlinear figure of merit in silicon nanowires integrated with 2D graphene oxide films", ACS Applied Materials and Interfaces 12 (29) 33094-33103 June 29 (2020). DOI:10.1021/acsami.0c07852.

[37] Linnan Jia, Dandan Cui, Jiayang Wu, Haifeng Feng, Tieshan Yang, Yunyi Yang, Yi Du, Weichang Hao, Baohua Jia, David J. Moss, "BiOBr nanoflakes with strong nonlinear optical properties towards hybrid integrated photonic devices", Applied Physics Letters Photonics 4090802 (2019). DOI: 10.1063/1.5116621

[38] Jiayang Wu, Yunyi Yang, Yang Qu, Xingyuan Xu, Yao Liang, Sai T. Chu, Brent E. Little, Roberto Morandotti, Baohua Jia, and David J. Moss, "Graphene oxide waveguide polarizers and polarization selective micro-ring resonators", Laser and Photonics Reviews 13 (9) 1900056 (2019). DOI:10.1002/lpor.201900056.

[39] Yunyi Yang, Jiayang Wu, Xingyuan Xu, Sai T. Chu, Brent E. Little, Roberto Morandotti, Baohua Jia, and David J. Moss, "Enhanced four-wave mixing in graphene oxide coated waveguides", Applied Physics Letters Photonics $\underline{3} 120803$ (2018); doi: 10.1063/1.5045509.

[40] Y. Qu, J. Wu, Y. Zhang, L. Jia, Y. Yang, X. Xu, S. T. Chu, B. E. Little, R. Morandotti, B. Jia, and D. J. Moss, "Graphene oxide for enhanced optical nonlinear performance in CMOS compatible integrated devices", Paper No. 11688-30, PW21O-OE109-36, 2D Photonic Materials and Devices IV, SPIE Photonics West, San Francisco CA March 6-11 (2021). doi.org/10.1117/12.2583978

[41] Yuning Zhang, Jiayang Wu, Yang Qu, Linnan Jia, Baohua Jia, and David J. Moss, "Optimizing the Kerr nonlinear optical performance of silicon waveguides integrated with 2D graphene oxide films", Journal of Lightwave Technology 39 Early Access (2021). DOI: 10.1109/JLT.2021.3069733

[42] L. Jia, J. Wu, T. Yang, B. Jia, D. J. Moss, "Large Third-Order Optical Nonlinearity in 2D PdSe2 Dichalcogenide Films for Nonlinear Photonic Devices", Paper No. 11689-34, PW21O-OE201-68, Integrated Optics: Devices, Materials, and Technologies XXV, SPIE Photonics West, San Francisco CA March 6-11 (2021). doi.org/10.1117/12.2584029

[43] H.Bao et al., "Laser Cavity Solitons and Turing Patterns in Microresonator Filtered Lasers: properties and perspectives", Paper No. LA203-5, Paper No. 11672-5, SPIE LASE, SPIE Photonics West, San Francisco CA March 6-11 (2021). doi.org/10.1117/12.2576645.

[44] Hamed Arianfard, Jiayang Wu, Saulius Juodkazis, David J. Moss, "Spectral Shaping Based via Integrated Coupled Sagnac Loop Reflectors in a Self-Coupled Nanowire", Preprints, DOI: 10.20944/preprints202104.0489.v1. 
[45] Hamed Arianfard, Jiayang Wu, Saulius Juodkazis and David J. Moss, "Three Waveguide Coupled Sagnac Loop Reflectors for Advanced Spectral Engineering", Journal of Lightwave Technology, vol. 39, Early Access (2021). DOI: 10.1109/JLT.2021.3066256.

[46] Yang Qu, Jiayang Wu, Yuning Zhang, Yao Liang, Baohua Jia, and David J. Moss, "Analysis of four-wave mixing in silicon nitride waveguides integrated with 2D layered graphene oxide films", Journal of Lightwave Technology, vol. 39, No. 9, 2902-2910 (2021). DOI: 10.1109/JLT.2021.3059721.

[47] Hamed Arianfard, Jiayang Wu, Saulius Juodkazis and David J. Moss, "Advanced Multi-Functional Integrated Photonic Filters based on Coupled Sagnac Loop Reflectors", Journal of Lightwave Technology, vol. 39, Issue: 5, pp.1400-1408 (2021). DOI:10.1109/JLT.2020.3037559. 